\title{
Oculodermatological findings in workers with occupational exposure to polychlorinated biphenyls (PCBs)
}

\author{
A FISCHBEIN,' JOAN N RIZZO,' S J SOLOMON, ${ }^{2}$ AND MARY S WOLFF'
}

From the Environmental Sciences Laboratory, ${ }^{1}$ Department of Community Medicine and Department of Neoplastic Diseases, ${ }^{2}$ Mount Sinai School of Medicine of the City University of New York, New York, New York 10029, USA

ABSTRACT Oculodermatological findings, such as hypersecretion of the Meibomian glands, swelling of the upper eyelids and hyperpigmentation of the conjunctivae are considered typical of ${ }^{\circ}$ "PCB poisoning." They were common clinical manifestations of Yusho and Yu-cheng, two

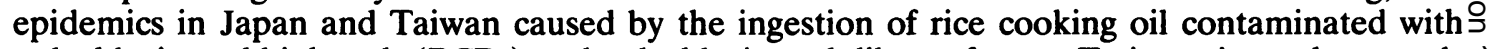
polychlorinated biphenyls (PCBs) and polychlorinated dibenzofurans. To investigate the preval- $\vec{c}$ ence of such abnormalities in a population with long term occupational exposure to PCBs, a $\subseteq$ group of 326 workers employed in two capacitor manufacturing facilities were investigated in ${ }^{\infty}$ 1976, and 195 of these again in 1979. The median blood values of lower homologues of PCBs $\ddot{\Phi}_{\mathscr{D}}$ were $63 \mathrm{ppb}$ (in plasma) in 1976 and $49 \mathrm{ppb}$ (in serum) in 1979, and of the higher homologues ${ }^{\top}$ $18 \mathrm{ppb}$ and $17.5 \mathrm{ppb}$ respectively. The prevalence of oculodermatological findings potentially related to the effects of PCBs were $9.4 \%$ and $13.3 \%$ at the two examinations. There was no significant association between such abnormalities and blood plasma/serum concentrations of PCBs. The observations in this work population exposed to PCBs differ from the Yusho and $\frac{\mathscr{Q}}{\varnothing}$ $\mathrm{Yu}$-cheng experiences in that fewer clinical abnormalities were found. Suggestions are made that $\stackrel{\bigcirc}{\circ}$ it may be inappropriate to extrapolate findings from the well known PCB poisoning episodes to $\overrightarrow{\overrightarrow{0}}$ exposures in occupational settings and that attention should be paid to the importance of polychlorinated dibenzofurans as an aetiological factor in human PCB poisoning.

Polychlorinated biphenyls (PCBs) are a group of complex chlorinated aromatic hydrocarbons that have been used industrially since the late 1920s. ${ }^{1}$ Because of their chemical stability, they were valuable for many industrial uses, such as additives to paints, surface coatings, and varnishes, and as a constituent in carbonless copy paper. Their principal use, however, has been as dielectric fluids in capacitors and transformers.

PCBs were manufactured in the United States under the trade name Aroclor ( $R$ ), and the numbers ascribed to the trade name identified the degree of chlorination. For exmaple, Aroclor 1254 and 1260 refer to $54 \%$ and $60 \%$ chlorine content respectively.

The manufacture of PCB containing carbonless copy paper was discontinued in the United States in

Received: 20 August 1984 Accepted: 1 October 1984
1971. In 1972 the Food and Drug Administration (FDA) established regulatory guidelines for the con trol of human exposure to PCBs after the com-응 pounds were shown to have contaminated food from packaging materials. ${ }^{2}$ The manufacturing of allo PCBs was discontinued in the United States in 1977> and their use further restricted. ${ }^{3}$

Despite the widespread industrial use of PCBs since the 1930s, it was not until 1966 that they were first recognised as a major environmental pollut- $N$ ant, ${ }^{45}$ and subsequently identified in the air, soil,,$心$ and water. Bioaccumulation has also been described in vegetation, fish, and milk products. ${ }^{6}$

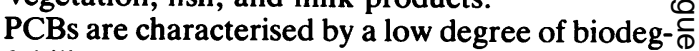
radability and tend to persist in the environment. $\stackrel{\oplus}{+}$ Levels of PCBs have been well documented in 0 people of industrialised nations. ${ }^{67}$ Since PCBs are ${ }^{\circ}$ lipophilic compounds, they tend to accumulate in tissues or organs according to adipose tissue content. $\stackrel{\overrightarrow{\mathrm{D}}}{\mathrm{Q}}$ 
Information available from numerous animal studies indicates a wide range of PCB related toxic effects on the liver, lipid metabolism, and both the immune and reproductive systems. ${ }^{89}$ PCBs produce hepatomas and hepatocellular carcinomas in experimental animals. ${ }^{1011}$ Differences between species in their response to PCBs have also been substantiated. ${ }^{812}$

Dermatological abnormalities have been frequently reported in animals exposed to PCBs. The pilosebaceous unit is the most commonly affected target organ, and acneiform lesions (chloracne) are considered typical sequelae from exposure to PCBs and related compounds. Enlargement of the Meibomian glands and swelling of the eyelids are also frequent manifestations associated with experimental PCB poisoning.

Human health effects associated with exposure to PCBs have not been well defined. In the 1930 s and 1940 s several investigators of occupationally exposed individuals reported multiple skin lesions characterised by chloracne, and occasionally associated with systemic effects. ${ }^{13}{ }^{14}$ It should be emphasised, however, that in many of the early reports exposures may well have been not only to PCBs but to a combination of PCBs and other halogenated hydrocarbons, particularly chlorinated naphthalenes. ${ }^{15}$

\section{Yusho and Yu-cheng}

A clinical syndrome of "PCB poisoning" was first described in detail in connection with two large scale outbreaks of PCB poisoning that were due to the ingestion of contaminated rice cooking oil. The first epidemic (Yusho: Japanese "oil disease") occurred in 1968 in Japan, and the second (Yu-cheng: Chinese "oil disease") in 1979 in Taiwan. ${ }^{16}{ }^{17}$ Chloracne, which is characterised by comedos, pustules, and straw coloured cysts was frequently observed. Oculodermatological signs and symptoms were important diagnostic criteria for these diseases and included hypersecretion of the Meibomian glands, swelling of the eyelids, and hyperpigmentation of the conjunctivae. These abnormalities were found in $60-85 \%$ of the affected individuals. ${ }^{18-20}$

It is important to note that polychlorinated dibenzofurans (PCDFs) and polychlorinated quaterphenyls (PCQs) were identified both in cooking oils and in patients with the Japanese and Taiwanese PCB poisoning and have been indicated as possible aetiological factors in the PCB poisoning. ${ }^{21-24}$

\section{Materials and methods}

To evaluate the health effects of long term occupa- tional exposure to PCBs, a cross sectional study was conducted in March 1976 of 326 workers employed in two capacitor manufacturing facilities. ${ }^{25}$ The use of PCB was discontinued in the manufacturing process in July 1977. A follow up examination was conducted 45 months later, in December 1979. Workers in PCB related occupations were invited to take part in the examinations on a voluntary basis. The most important criterion for inclusion in the study was long term employment of 20 years or more, although some individuals with shorter durations of employment who came to the examination were also included. The exposure of all individuals to PCBs occurred during employment in the production of capacitors, which were filled with dielectric fluid containing PCBs.

There was a potential for exposure through skin absorption, inhalation, and, to a lesser degree, ingestion of fluid containing PCBs. The workers handled heated and partially volatilised dielectric fluids. A wide range of laboratory and clinical tests was performed on the study population, and various questionnaires were administered to determine occupational and medical histories, and to elicit symptoms potentially related to the toxic effects of PCBs. Specially designed forms were used to record oculodermatological findings. PCBs were measured in plasma and serum by gas chromatography (electron capture detection) according to methods previously described. ${ }^{26}$ PCB residues were calculated as Aroclor 1248 (for peaks with retention times less than p,p'-DDE) and designated as lower homologues of PCBs (LPCB). Peaks with retention times greater than p,p'-DDE were calculated as Aroclor 1254, and regarded as higher homologues of PCBs (HPCB). A clinical biochemistry screen was also performed and included liver function tests (SGOT, SGPT, Alk phos, $\gamma$-GTP, LDH, and bilirubin) and serum lipids (triglycerides and cholesterol).

PCB levels were available in 289 of the 326 $(88.7 \%)$ subjects in 1976 . To avoid potentially confounding factors in the analysis of the data, 43 subjects with a history of excessive alcohol consumption or of taking medications with potential effects on the liver and serum lipids were excluded from the analysis. The study sample thus consisted of 246 individuals in 1976, 106 women aged 23 to 60 (median age 49), and 140 men aged 21 to 67 (median age 40). Although there was a significant difference in age between the sexes (Wilcoxon two sample tests, $s=1569.5, p<\cdot 0001)$, this pattern was not observed for any of the other independent variables of interest. The individuals were subdivided into two groups based on the presence or absence of oculodermatological abnormalities considered to be related to PCBs. Twenty three indi- 
viduals ( 9 women and 14 men) were classified in the former category. For the analysis of change in PCB levels between the two examinations, paired data were available on 101 subjects.

The data were encoded on computer compatible forms and entered into an IBM 370 computer for analysis using primarily the Statistical Analysis System (SAS). The univariate statistics for each variable were generated, and the nature of the statistical distributions were evaluated for normality using the Kolmogrov-Smirnov maximum deviation statistic (D). Total PCBs (TPCB), lower chlorinated PCBs (LPCB), and higher chlorinated PCBs (HPCB) were normalised by logarithmic transformations of the data. The transformed values were used in all inferential parametric statistical tests.

Group differences were evaluated by computing $t$ tests with respect to the following parameters: SGOT, SGPT, $\gamma$-GTP, Alk phos, LDH, bilirubin, triglycerides, cholesterol, TPCBs, HPCBs, and LPCBs, and both systolic and diastolic blood pressures.

\section{Results}

The median LPCB values of the examined workers were $63 \mathrm{ppb}$ in 1976 and $49 \mathrm{ppb}$ in 1979 , whereas the HPCB levels were $18 \mathrm{ppb}$ and $17.5 \mathrm{ppb}$. Although the mean HPCB levels of the exposed workers did not decrease from the time of the initial study $(t=-0.46, p>0.05)$, there was a significant reduction in LPCB $(t=-2 \cdot 66, p<0.008)$.

The table shows the frequency of oculodermatological abnormalities in the examined population. At the first examination, 29 workers (9.4\%) had at least one oculodermatological sign and five individuals had more than one abnormality. Seventeen $(5.5 \%)$ had injected conjunctiva, seven $(2.3 \%)$ had eye discharge, five $(1.6 \%)$ had swelling of the upper eyelid, and four $(1 \cdot 3 \%)$ had enlarged Meibo- mian glands.

In the follow up study performed in 1979, 195 of the original 326 workers returned for reexamination. Fourteen were excluded from the $\overrightarrow{\vec{\sim}}$ analysis due to "other" abnormalities as in the table:0 Of the remaining $181,24(13.3 \%)$ exhibited abnormal oculodermatological findings. Fifteen $\overline{\bar{c}}$. $(8.3 \%)$ had injected conjunctiva, three $(1.7 \%) \widetilde{\curvearrowright}$ showed discharge, four $(2.2 \%)$ eyelid oedema, and two $(1 \cdot 1 \%)$ had enlarged Meibomian glands. None ${ }^{\infty}$ had abnormal pigmentation of the conjunctiva, and $\overrightarrow{0}$ of those with injected conjunctiva, only one had had $-\overrightarrow{-}$ this abnormality in 1976. Eleven individuals $\vec{o}^{-}$ categorised as "injected" at the second examinationo had been classified as normal in 1976, and one had had eyelid oedema in 1976. Two workers who weree excluded from consideration in 1976 because of "other" or pupillary abnormality had injected con-t junctiva in 1979.

Significant differences were not found for PCBP levels, liver function tests, serum lipids, or systolic and diastolic blood pressures between those with of without oculodermatological signs. All mean levels were within the normal laboratory range. The low prevalence of abnormal clinical biochemistry test have been summarised previously. ${ }^{25}$

\section{Discussion}

In the major outbreaks of PCB poisoning from ingestion of contaminated oil in Japan and Taiwan most $(60 \%-85 \%)$ exposed individuals developect oculodermatological signs, ${ }^{19} 20$ characterised by swel 3 ling of the upper eyelid, enlargement of the Meibo mian glands with eye discharge, hyperpigmentationg. and injection of the conjunctiva. At the time these were regarded as typical PCB related abnormalities $\frac{\mathbb{D}}{3}$

In the present investigation we examined a group of workers with long term occupational exposure to. PCBs with $30-60 \%$ chlorination, and significang

Oculodermatological abnormalities in PCB exposed workers by comparison with patients with Yusho and Yu-cheng

\begin{tabular}{|c|c|c|c|c|}
\hline \multirow[t]{2}{*}{ Conditions* } & \multicolumn{2}{|l|}{ PCB exposed workers } & \multirow{2}{*}{$\begin{array}{l}\text { Yusho } \$ \text { patients } \\
(n=189) \\
\text { (\%) }\end{array}$} & \multirow{2}{*}{$\begin{array}{l}\text { Yu-cheng } \S \text { patients } \\
(n=130) \\
(\%)\end{array}$} \\
\hline & $\begin{array}{l}1 \text { st } \operatorname{exam}\left(n=310^{*}\right) \\
\text { No }(\%)\end{array}$ & $\begin{array}{l}\text { 2nd exam }(n=181 \ddagger) \\
\text { No }(\%)\end{array}$ & & \\
\hline $\begin{array}{l}\text { Oedema of upper eyelid } \\
\text { Injected conjunctiva } \\
\text { Eye discharge } \\
\text { Hyperpigmentation } \\
\text { Enlargement of Meibomian glands } \\
\text { Total }\end{array}$ & $\begin{aligned} 5(1 \cdot 6) \\
17(5 \cdot 5) \\
7(2 \cdot 3) \\
4(1 \cdot 3) \\
4(1 \cdot 3) \\
37+(12 \cdot 0)\end{aligned}$ & $\begin{array}{l}4(2 \cdot 2) \\
15(8 \cdot 3) \\
3(1 \cdot 7) \\
0(0 \cdot 0) \\
2(1 \cdot 1) \\
24(13 \cdot 3)\end{array}$ & $\begin{array}{l}(73 \cdot 0) \\
(70 \cdot 8) \\
(85 \cdot 7) \\
= \\
=\end{array}$ & $\begin{array}{l}(58 \cdot 9) \\
(60 \cdot 1) \\
(80 \cdot 5) \\
(66 \cdot 6) \\
(70 \cdot 1)\end{array}$ \\
\hline
\end{tabular}

*Eleven individuals were excluded with "other" ocular findings, such as pterygium (5), pallor (4), blepharitis (1), and thin eyebrows (1): These signs were not thought to be related to PCB poisoning. Four patients had post-traumatic pupillary changes and were excluded as well.

†Five patients had multiple ocular abnormalities. Total number of patients with oculodermatological abnormalities was 29.

$\ddagger$ Two individuals were excluded because of unavailable data, eight had other ocular findings, whereas four had abnormal pupils.

§Figures are approximate values based on discrepancies in reporting. 1420 
absorption of PCBs was reflected in the high serum PCB levels. By contrast, the PCB levels in patients with Yusho and Yu-cheng were generally lower than those found in the present study, although methodological variations may account for some of the differences. ${ }^{27}$

Our observations show a discrepancy in the clinical effects reported for the Yusho and Yu-cheng patients and our occupationally exposed study group-that is, despite higher PCB levels among the workers, the prevalence of oculodermatological signs was much lower than in the typical Yusho and Yu-cheng patients. This is consistent with our previously reported findings concerning dermatological abnormalities, including chloracne. ${ }^{28}$

In evaluating these differences it should be recognised that several investigators have identified polychlorinated dibenzofurans and polychlorinated quaterphenyls in the contaminated cooking oils, as well as in the patients with Yusho and Yucheng. ${ }^{21-24} 2930$ It has also been shown that PCDFs are relatively more toxic than PCBs. ${ }^{31}{ }^{32}$ Moreover, it has been further suggested that these contaminants may have played a crucial part in the appearance of the toxic clinical signs of PCB poisoning. ${ }^{30}$ Different toxic responses observed in animal studies have also been attributed to contamination of some PCB mixtures with PCDFs and perhaps chlorinated naphthalenes. ${ }^{31}$

At present, we do not have conclusive evidence concerning PCDFs in the dielectric fluid to which our study subjects were exposed. Elsewhere, analyses of PCBs of varying degrees of chlorination have shown levels of PCDFs less than $1 / 1000$ as compared with those found in Yusho and Yu-cheng. ${ }^{33}$

Another possible explanation for the apparent discrepancy in clinical findings may relate to the route of exposure. Our subjects were exposed in PCBs in an occupational setting, in which long term exposure to volatilised PCBs occurred as well as exposure by skin contact; by contrast, the intoxication in Japan and Taiwan resulted from the ingestion of contaminated oil over a relatively short time. Our study suggests that it may be inappropriate (with the present knowledge of PCB related compounds) to extrapolate from Yusho and Yu-cheng any clinical effects that might be related to PCB exposure in an occupational environment. Based on our investigation, severe clinical effects are relatively rare and differ considerably in degree from the Japanese and Taiwanese experiences. The current lack of corroborative data from analytical chemistry to support the presence of PCDFs and PCQs in the dielectric fluid and in the exposed workers prevents us at this time from reaching final conclusions about causeeffect relations.
This work was supported by the National Institute of Environmental Health Sciences, Center Grant No ES 00928, and by a grant from the General Electric Company.

We are grateful to Mr Sibel for his skilful secretarial help in the typing of this manuscript.

\section{References}

' Hutzinger O, Safe S, Zitko V. The chemistry of PCBs. Cleveland, Ohio: CRC Press, 1974.

${ }^{2}$ US Federal Register. Part II 1973;38(129).

${ }^{3}$ Environmental Protection Agency. Polyychlorinated biphenyls (PCBs); manufacturing processing, distribution in commerce and use prohibition; use in closed and controlled waste manufacturing processes. Federal Register 1982;47(204):4698096.

4 Anonymous. Report of a new chemical hazard. New Scientist 1966;32:612.

5 Jensen S. The PCB story. Ambio 1972;1:123-31.

- Committee on the Assessment of Polychlorinated Biphenyls in the Environment, Environmental Studies Board, Commission on Natural Resources, National Research Council. Polychlorinated biphenyls. Washington, DC: National Academy of Sciences, 1979.

${ }^{7}$ Finklea JL, Priester JR, Creason T, Hauser T, Hinners T, Hammer DI. Polychlorinated biphenyl residues in human plasma expose a major urban population problem. Am J Public Health 1972;62:645-51.

${ }^{8}$ Allen JR. Response of the non-human primate to PCB exposure. Fed Proc 1975;34:1965-79.

${ }^{9}$ McConnell E, Hass JR, Altman N, Moore JA. A spontaneous outbreak of polychlorinated biphenyl (PCB) toxicity in Rhesus monkeys (Macaca mulatta): toxicopathology. Laboratory Animal Science 1979;29:666-73.

${ }^{10}$ Kimbrough RD, Linder RE. Induction of andenofibrosis and hepatomas of the liver of Balb/cd mice by polychlorinated biphenyls (Aroclor 1254). J Natl Cancer Inst 1974;53:547-52.

" Kimbrough RD, Squire R, Linder RE, Strandberg JD, Montali RJ, Burse VW. Induction of liver tumors in Sherman strain female rats by polychlorinated biphenyl (Aroclor 1260). J Natl Cancer Inst 1975;55:1453-9.

12 Koller LD, Zinkl JG. Pathology of polychlorinated bi-phenyls in rabbits. Am J Pathol 1973;70:363-78.

${ }^{13}$ Schwartz L. Dermatitis from synthetic resins and waxes. Am J Public Health 1936;26:586-92.

14 Good CM, Pensky N. Halowax acne ("cable rash"): a cutaneous eruption in marine electricians due to certain chlorinated naphthalenes and diphenyls. Archives of Dermatology and Syphilology 1943;48:251-7.

is Schwartz L. An outbreak of halowax acne ("cable rash") among electricians. JAMA 1943; 122:158-61.

${ }^{16}$ Higuchi K, ed. PCB poisoning and pollution. Tokyo: Kodansha, Ltd, New York: Academic Press, 1976.

${ }^{17}$ Hsu S-T, Ma C-I, Hsu SK-H, Wu S-S, Hsu N H-M, Yeh C-C. Discovery and epidemiology of PCB poisoning in Taiwan. Am J Ind Med 1984;5:71-9.

${ }^{18}$ Ohumaru M. Past and present medical states of Yusho patients. Am J Ind Med 1984;5:13-8.

19 Ikui H, Sugi K, Uga S. Ocular signs of chronic chlorobiphenyls poisoning ("Yusho"). Fukuoka Acta Medica 1969;60:432-9.

${ }^{20} \mathrm{Fu}$ Y-A. Ocular manifestation of polychlorinated biphenyls intoxication. Am J Ind Med 1984;5:127-32.

${ }^{21}$ Miyata H, Murakami Y, Kashimoto T. Determination of polychlorinated quaterphenyl (PCQ) in Kanemi rice oil which 
caused "Yusho" and investigation on the PCQ formation. Journal of the Food Hygiene Society Japan 1978;19:417-25.

${ }^{22}$ Masuda Y, Yoshimura H. Polychlorinated biphenyls and dibenzofurans in patients with Yusho and their toxicological significance. Am J Ind Med 1984;5:31-44.

${ }^{23}$ Kashimoto T, Miyata H, Kunita N. The presence of polychlorinated quaterphenyls in the tissues of Yusho victims. Fd Cosmet Toxicol 1981;19:335-40.

${ }^{24}$ Chen PH-S, Luo M-L, Wong C-K, Chen C-J. Polychlorinated biphenyls, dibenzofurans, and quaterphenyls in the toxic ricebran oil and PCBs in the blood of patients with PCB poisoning in Taiwan. Am J Ind Med 1984;5:133-45.

${ }^{25}$ Fischbein A, Wolff MS, Lilis R, Thornton J, Selikoff IJ. Clinical findings among PCB-exposed capacitor manufacturing workers. Ann NY Acad Sci 1979;320:703-15.

${ }^{26}$ Wolff MS, Fischbein A, Thornton J, Rice C, Lilis R, Selikoff IJ. Body burden of polychlorinated biphenyls among persons employed in capacitor manufacturing. Int Arch Occup Environ Health 1982; 49:199-208.

${ }^{27}$ Kunita N, Kashimoto T, Miyata H, Fukushuma A, Hori S, Obana H. Causal agents of Yusho. Am J Ind Med 1984;5:4558 .

${ }^{28}$ Fischbein A, Wolff MS, Bernstein J, Selikoff IJ. Dermatological findings in capacitor manufacturing workers exposed to dielec- $\longrightarrow$ tric fluids containing polychlorinated biphenyls (PCBs). Arch Environ Health 1982;37:69-74.

${ }^{24}$ Kuratsune M. Yusho. In: Kimbrough RD, ed. Halogenated biphenyls, terphenyls, naphthalenes, dibenzodioxins and related $\stackrel{\mathcal{+}}{+}$ products. Amsterdam: Elsevier/North Holland BiomedicalO Press 1980:287-302.

${ }^{30}$ Kashimoto T, Miyata $\mathrm{H}$, Kunita S, et al. Role of polychlorinated $\overline{\bar{C}}$ dibenzofuran in Yusho (PCB poisoning). Arch Environ Health $\vec{\Phi}$ 1981;36:321-6.

${ }^{31}$ Vos JG, Koeman JH, van der Maas HL, Ten Noever de Brauwd MC, deVos RH. Identification and toxicological evaluation of chlorinated dibenzofuran and chlorinated naphthalene in two $\vec{\circ}$ commercial polychlorinated biphenyls. Fd Cosmet Toxicol1970;8:625-33.

${ }^{32}$ Yoshihara S, Nagata K, Yoshimura H, Kuroki H, Masuda Y. Inductive effect on hepatic enzymes and acute toxicity of indi- $O$ vidual polychlorinated dibenzofuran congeners in rats. Toxicol Appl Pharmacol 1981;59:580-8.

${ }^{33}$ Morita M, Nakagawa J, Akiyama K, Mimura S, Isono N. N Detailed examination of polychlorinated dibenzofurans inos PCB preparations and Kanemi Yusho oil. Bull Environ Contam Toxicol 1977;18:67-73. 